\title{
A high temperature apparatus for measurement of the Seebeck coefficient
}

\author{
Shiho Iwanaga, Eric S. Toberer, Aaron LaLonde, and G. Jeffrey Snyder \\ Materials Science, California Institute of Technology, Pasadena, California 91125, USA
}

(Received 18 March 2011; accepted 25 May 2011; published online 30 June 2011)

\begin{abstract}
A high temperature Seebeck coefficient measurement apparatus with various features to minimize typical sources of error is designed and built. Common sources of temperature and voltage measurement error are described and principles to overcome these are proposed. With these guiding principles, a high temperature Seebeck measurement apparatus with a uniaxial 4-point contact geometry is designed to operate from room temperature to over $1200 \mathrm{~K}$. This instrument design is simple to operate, and suitable for bulk samples with a broad range of physical types and shapes. (c) 2011 American Institute of Physics. [doi:10.1063/1.3601358]
\end{abstract}

\section{INTRODUCTION}

The Seebeck coefficient is an intrinsic material property which describes the voltage $(\Delta V)$ that develops from a given temperature difference $(\Delta T)$ in a material $(S=\Delta V / \Delta T)$. The Seebeck coefficient is affected by the transport properties of charge carriers and is thus sensitive to impurities, defects, and phase transformations in materials. As such, it is studied in fields of research including superconductors, ${ }^{1,2}$ solid state fuel cell electrolytes, ${ }^{3}$ and thermoelectrics. ${ }^{4}$ In thermoelectric devices, the Seebeck coefficient is an integral part the heat-toelectricity conversion and the figure-of-merit of a thermoelectric material is given by $z T=S^{2} \sigma T / \kappa$, where $S$ is the Seebeck coefficient, $\sigma$ is the electrical conductivity, $\kappa$ is the thermal conductivity, and $T$ is the temperature.

Seebeck coefficient measurements above room temperature are critical for the development and testing of high temperature thermoelectric generators. Thermoelectric power generation has traditionally been used as a power source in remote locations; a more recent interest is the utilization of thermoelectric devices for wasted heat recovery, making accurate measurement of the Seebeck coefficient particularly critical for renewable energy applications. The measurement of the Seebeck coefficient at high temperatures presents many challenges and the associated history and developments on this topic has recently been reviewed. ${ }^{5}$

High temperature Seebeck measurements are often subject to irreproducibility and inconsistency in results due to a lack of standardized guidelines for the measurement procedure. ${ }^{5,6}$ For example, errors in temperature measurement occur in the high temperature range due to poor thermal contact between the thermocouple and the sample surface, ${ }^{6}$ leading to considerable variation of results between laboratories. In this paper, we describe the most commonly used Seebeck coefficient measurement techniques and the associated potential drawbacks between methods. Additionally, principles to aid the development of improved apparatuses are proposed and the construction of a specific device is described.

\section{A. Seebeck measurement and assumptions}

In principle, the measurement of the Seebeck coefficient in bulk materials is relatively simple. It is only necessary to know the temperature difference between two locations on a sample, and the voltage across the two points. Figure 1 shows the ideal measurement geometry where two probes make a point contact with the material. The probes dually serve as the thermocouples and the voltage leads, across which the temperature difference and associated voltage is measured. The implicit assumptions in such a measurement include (a) the system is in steady state during the measurement of the temperature and voltage, and that both measurements occur simultaneously, (b) the voltage response to the temperature gradient is linear, and (c) the measurement of the temperature and voltage occurs at the same point on the sample.

In a real instrument, however, non-negligible errors are often present due to the inability to achieve the assumed conditions. For assumption (a), temperature and voltage measurements occur at different times in practice, and the need for simultaneous measurement is well described in the review by Martin. ${ }^{5}$ The assumption (b) can fail in practice due to insufficient signal or non-zero voltage at $\Delta T=0$. During high temperature measurements, there is often a noticeable voltage offset, ${ }^{5}$ which can ranges from few $\mu \mathrm{V}$ due to the electronics to almost $1 \mathrm{mV}$, which can sometimes be observed at high temperatures. The origin of the large offset and its effect on the accuracy of the measured Seebeck coefficient is unknown. The last assumption in (c) is never entirely accurate, as there is always a definite distance between the temperature and voltage measurement locations due to the finite size of the temperature sensor. The smallest point source temperature sensor used in Seebeck measurements is a thermocouple. In a thermocouple junction, the temperature where the wires meet is not necessarily the same as the sample temperature associated with the voltage measurement.

\section{B. Typical measurement geometries}

While all instrument setups deviate from the ideal measurement conditions, certain designs are less susceptible to error than others. Currently, there are two dominant measurement geometries, as shown in Figs. 2(a) and 2(b). In the first geometry in Fig. 2(a), a bulk sample is placed between two metal blocks, which act as a heat source and sink. In this setup, the thermocouples are embedded in the metal blocks 


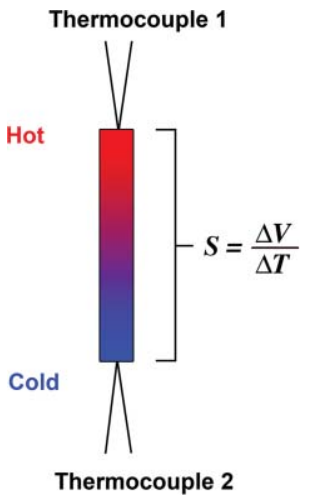

FIG. 1. (Color online) Ideal Seebeck coefficient measurement geometry, with thermocouples making small point contacts.

rather than directly contacting the sample. This setup gives the advantage of avoiding chemical reactions between the sample and the thermocouple materials (which will be discussed later) and has few sample geometry restrictions. However, this system inherently has thermal and electrical contact resistance in these metal blocks themselves as well as across the interfaces between the sample and the metal blocks. In particular, the thermal contact resistances may lead to offsets in the temperature measurement.

In the second case shown in Fig. 2(b), a long bar-shaped sample is placed between the heat source and sink. The improvement of the Fig. 2(b) geometry over Fig. 2(a) can be understood by analogy to 2- and 4-point electrical resistance measurements. When measuring materials with low electrical impedance, 4-point measurements are necessary to eliminate the contact resistance errors. Likewise, a 4-point thermal design reduces the effect of thermal contact resistance. ${ }^{5}$ Such a design is shown in Fig. 2(b), where the key difference from Fig. 2(a) is the placement of the thermocouples along the side of the sample. While this important modification eliminates the thermal contact resistance between the sample and the heat source/sink, several issues remain. The first issue is at high temperatures, where the thermal conductance of the thermocouples can draw heat away from (a)

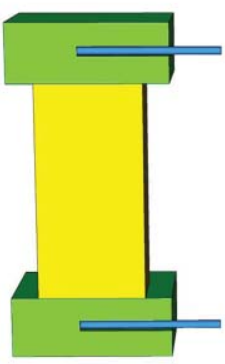

(b)

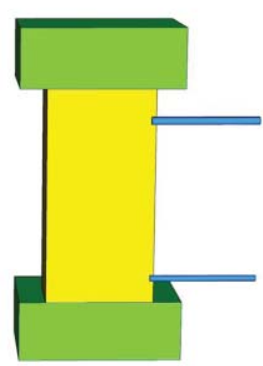

(c)

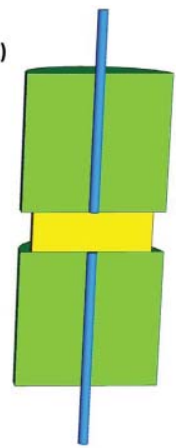

FIG. 2. (Color online) Three general geometries for measurement of the Seebeck coefficient, shown in cross-sectional view. (a) 2-point geometry where thermocouples are embedded in heater blocks, (b) off-axis 4-point method where thermocouples contact the side of the sample, and (c) our proposed uniaxial 4-point method. The upper and lower green blocks represent heaters and/or heat sinks, the center yellow block the bulk sample, and blue narrow rods the thermocouples.

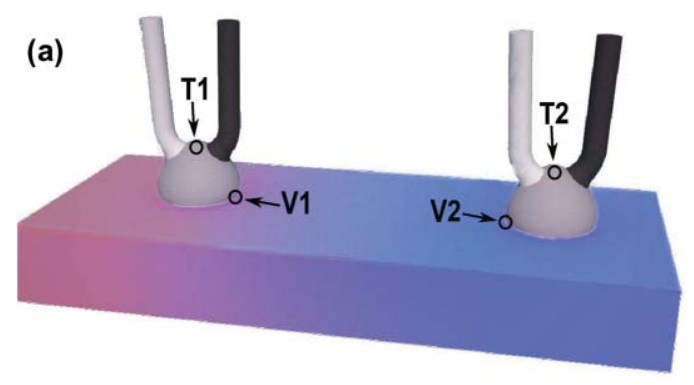

(b)

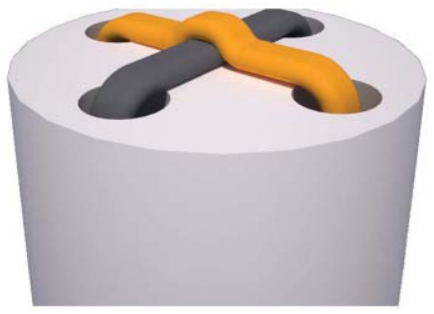

FIG. 3. (Color online) Thermocouple probes used for Seebeck measurement. (a) Two probes made from thermocouples with beads. The Seebeck voltage produced in the sample (V1 and V2) do not necessary occur at the same location as the associated temperature measurements (T1 and T2). This spatial variation can lead to errors when thermal gradients are present across the thermocouple bead and across the area in contact with the sample. (b) To minimize these errors, a crossed-wire geometry is utilized on a isothermal surface of the sample. Extremely fine wires are threaded through a 4-bore ceramic tube and mechanical force is used to ensure good electrical and thermal contact.

the sample (known as the "cold-finger effect") leading to a temperature difference across the thermocouple bead. This effect will result in the voltage and temperature measurements being made at locations with different temperatures (Fig. 3(a)). Second, lateral spring loading is required to ensure good thermal contact between the thermocouples and the sample surface. At high temperatures, plastic deformation often occurs in soft samples and can lead to poor contacts. In brittle samples, side loading can lead to sample fracture or breakage. In an attempt to accommodate the side loading issues, low spring forces are used, however, this can lead to high contact resistance between the thermocouple and the sample. A third issue is the uncertainty in the temperature reading, as the thermocouple junction is contacting a sample surface with an ill-defined temperature because it is in a temperature gradient. The geometry in Fig. 2(b) uses an elongated geometry in an effort to reduce $d T / d z$ across the thermocouple-sample junctions. Shaping the sample into an elongated geometry may be challenging since good thermoelectric materials are often brittle and can be easily damaged during cutting. Further, this geometry is unsuitable for flash diffusivity measurements, often leading to the use of more than one sample to acquire the properties of interest for full characterization.

\section{PROPOSED INSTRUMENT GEOMETRY AND MEASUREMENT PROCEDURES}

\section{A. Instrument geometry}

A schematic of the proposed instrument geometry that solves some of common problems described in Sec. I B is 
shown in Fig. 2(c). The uniaxial 4-point Seebeck system concept originates from NASA-JPL in the 1980s during the development of an apparatus using a light pipe to supply a dynamic $\Delta T .^{7}$ The system described below in detail encompasses several differences from the light pipe system but the geometry is generally the same. The main advantages of this geometry are: (1) the thermocouples make direct contact to the sample surface, (2) the thermocouple junctions contact an isothermal temperature surface, and (3) the uniaxial design allows thermocouples to exert larger forces onto the sample surface, contributing to the minimization of the thermal and electrical contact resistance.

In the current design, the cylindrical heater element, made from machinable boron nitride, has a through-hole at the center in which the thermocouple goes through. The thermocouple, as will be discussed in detail later, is made from a 4-bore ceramic tube, and has a slightly smaller diameter compared to the diameter of the hole in the heater element. This design allows the thermocouple to contact directly with the sample surface independently of the heater cylinder which heats the sample surface by conduction. In this design, the thermocouple ceramic tube is heat-sunk to the heaters, thereby reducing cold-finger effects. This configuration is in contrast to the off-axis design (Fig. 2(b)), where the cold finger effect is exacerbated by the low thermocouple-sample contact resistance and the temperature difference between the sample and ambient temperature.

The second advantage, illustrated in Fig. 2(c), is the thermocouple contact to a constant temperature region on the sample surface created by the intimate contact between the heater and the sample. In each of the geometries shown in Fig. 2, the heaters establish a temperature gradient along the principle axis. The finite size of the thermocouple bead in the off-axis geometry (Fig. 2(b)) is only able to contact the sample surface across the temperature gradient that exists at the contact area. Only the proposed design in Fig. 2(c) allows the thermocouple junction to make contact with an isothermal surface. Additionally, the proposed arrangement of the heaters and thermocouples, like the arrangement in Fig. 2(a), allows for a wide range of sample shapes and sizes to be measured. Typical sample fabrication methods, such as hot pressing or solidification, result in cylindrical samples that can be easily cut into a disk shaped sample. Having the Seebeck measurement system capable of measuring disk shaped samples is beneficial as the geometry is suitable for resistivity, Hall effect using Van der Pauw geometry, and thermal diffusivity measurements. Further, the uniaxial 4-point geometry measures the full $\Delta T$ across the sample providing maximized voltage signals for measurement of thinner samples.

The third advantage of the proposed design is the application of the compressive forces by springs along a single principle axis (uniaxial design), ensuring good thermomechanical contacts. The springs attached to the heater assembly are designed to exert a uniform force onto the sample for good contact between the heaters and the sample surfaces. The compression springs on the thermocouples, which are independent from the heater springs, provide pressure to the thermocouple junction at the sample surface.
It is noted that, heaters and thermocouples exerting forces onto the sample surfaces by independent compression is one of the major differences from the light pipe design. The uniaxial design is attractive for both brittle and soft materials as there are no tensile stresses present to promote crack propagation.

\section{B. Thermocouple design}

In concert with system-level design advancements, there are specific thermocouple designs that can improve temperature and voltage measurements. Typical temperature measurements in high temperature environment use thermocouple types which contain platinum wires, such as type $\mathrm{S}$ or R. In our embodiment, a homemade thermocouple type composed of niobium and chromel wires is used and can sustain up to $\sim 925 \mathrm{~K}$. There are multiple reasons to use this non-standard thermocouple type for Seebeck measurements.

First, as briefly mentioned in Sec. I B, a chemical reaction between the thermocouple and the sample frequently occurs at the contact point between the two and leads to errors in the measured Seebeck slope and voltage offset. In order to minimize this effect, thermocouples with minimal reactivity with the sample should be chosen. Platinum thermocouples, while resistant to oxidation, are rather reactive to heavy metals such as $\mathrm{Pb}, \mathrm{Te}, \mathrm{Ag}, \mathrm{Bi}$, and $\mathrm{Sb}$ which are common in thermoelectric materials. Even traditionally nonreactive nickel has some reactivity with certain thermoelectric elements at high temperatures. Therefore, thermocouple wires that are inert to heavy metals are desired.

Traditional Seebeck systems use one of the thermocouple wires for the voltage measurement. If there is a temperature difference between the point of the voltage and temperature measurements (typically at least across the diameter of the wire used for voltage measurement) a voltage error will arise. The error induced by this factor is very apparent when measuring low Seebeck coefficient materials with high thermal conductivity (e.g., tungsten). To reduce this effect, a near-zero Seebeck coefficient metal, such as copper or niobium should be used for the voltage probe (closest to the sample) in combination with a high Seebeck material for making the thermocouple. While the resulting thermocouple supplies only half the voltage of traditional thermocouples, measurement of this reduced signal is well within the capabilities of modern electronics.

For high temperatures, tungsten and niobium have been found to be preferable due to their low reactivity. In our current setup, the combination of the niobium and chromel is used. Minor drawbacks for these elements are that they must be used in an oxygen free environment and are poor thermocouples for near-room temperature operation. It is noted that other combinations of thermocouples are possible: niobium/tungsten (up to above $1000^{\circ} \mathrm{C}$ ), niobium/tungsten-rhenium, copper-constantan (type $\mathrm{T}$ ) and gold-iron/chromel.

In order to provide an additional barrier against chemical reaction, thin and flexible Grafoil sheets (polycrystalline graphite foil, for example, from GrafTech International 
Inc, Ohio) are frequently used between the interface of the sample and thermocouple. With high thermal conductivity $\left(\sim 5 \mathrm{~W} / \mathrm{mK}\right.$ at room temperature $\left.{ }^{8}\right)$ and low electrical resistivity ( $\sim 1.15 \mathrm{~m} \Omega \mathrm{m}$ at room temperature), these sheets additionally provide good thermal contact between the sample and the heating blocks while not interfering with the voltage measurements.

Large thermocouple junctions risk reduced accuracy in the surface temperature measurement because of the temperature differences between the points of voltage and temperature measurement. Figure 3(a) shows a schematic of a large beaded junction is often used in many instruments. In this case, the Seebeck voltage measurement (between V1 and V2) does not necessary occur where temperatures (T1 and T2) are measured. It is advised that having low pressurized inert gas such as argon or helium gas may aid the heat transfer by convection.

One drawback of having a small beaded junction is that it suffers from poor mechanical strength. Forming the bead frequently embrittles the junction making the wires more difficult to handle and easier to break or otherwise damage. We have found that having a "crossed wire" junction instead of a bead welded junction provides less of this mechanical problem. This design is advantageous as it avoids the alloying and embrittlement associated with welding beads. We currently utilize wires with a diameter of 0.005 inches (or $\sim 125 \mu \mathrm{m}$ ) but other sizes have been used successfully. If a thin soft wire (such as copper) is mated with a hard wire, the soft wire may eventually break after many uses.

Figure 3(b) shows the "cross" geometry, where wires threaded down a 4-bore ceramic tube are made to cross. Here, the contact between the wires is mechanical and governed by the geometry of the crossed wires. To further ensure that the junction is near isothermal, the ceramic tubing used to house the wires is made of low thermal conductivity mullite (1/16 inch in diameter). While such a thin mullite tube is delicate, the force loading is strictly compressive and along the length of the tube. The ability to simply and rapidly develop new thermocouple material combinations is a final advantage delivered by this geometry.

\section{Measurement procedure}

Beyond fundamental changes in geometry, other design features are implemented to improve accuracy in steady-state measurements of the Seebeck coefficient. As discussed in Sec. I A, steady state measurements ideally have all measurements occur simultaneously. In practice, measurements of temperature and voltage are often conducted sequentially. A first-order correction to thermal drift is to use the delta method, where measurements are symmetric with time. For example, measurements taken in the order $T_{c 1}, T_{h 1}, \Delta V, T_{h 2}$, $T_{c 2}$ at equally spaced intervals in time account for linear drift when $T_{c}=\left(T_{c 1}+T_{c 2}\right) / 2$ and $T_{h}=\left(T_{h 1}+T_{h 2}\right) / 2$.

The absolute Seebeck coefficient is corrected from the contribution of the voltage contact wires by subtracting off the Seebeck voltage that these wires produce (typically $\mathrm{Cu}$, $\mathrm{Nb}, \mathrm{Pt}$, or $\mathrm{W}$ ). The absolute Seebeck coefficient for $\mathrm{Cu}, \mathrm{Pt}$, and $\mathrm{W}$ are given by Roberts ${ }^{9,10}$ and the Seebeck of Nb is reported by Burkov. ${ }^{11}$ The thermoelectric voltages of common thermocouple wires relative to Pt were measured by NIST and reported in NIST Monograph $175 .^{12}$

\section{Slope method}

The Seebeck coefficient can be found from the slope of the $\Delta V$ vs. $\Delta T$ as discussed in Sec. I, and the multiple gradient method ${ }^{13}$ is implemented here. In this method, the sample temperatures are controlled so that $\Delta T$ values across the sample can be adjusted continuously from positive to negative. The corresponding $\Delta V$ values are measured and plotted which allows any offset voltage to be eliminated. ${ }^{6}$

In our setup, the temperatures of the top heater and the bottom heater are controlled so that $T_{h}$ and $T_{c}$ values oscillate around one value of the temperature, which we use for the average temperature of the sample. In this oscillation mode, the maximum peak-to-peak $\Delta T$ of usually about $10-20^{\circ} \mathrm{C}$ are applied. It is noted that, non-linear fitting of the $\Delta V$ vs. $\Delta T$ plot can be indicative of poor signal-to-noise ratio, requiring larger $\Delta T$. A $\Delta T$ of $3 \%$ of the absolute sample temperature is often appropriate.

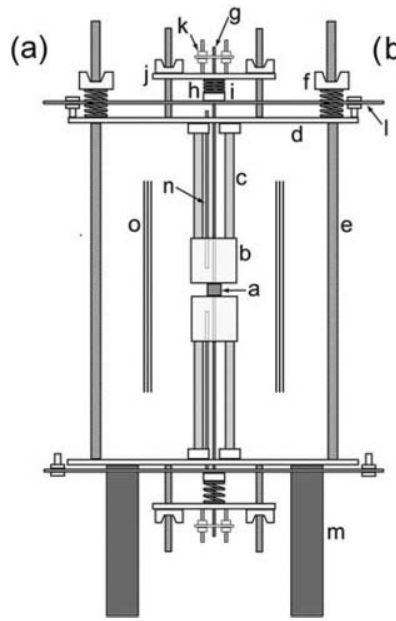

(b)

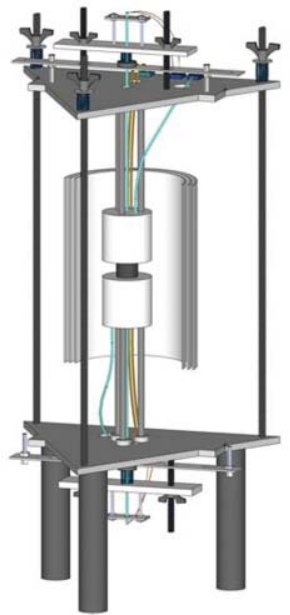

(c)

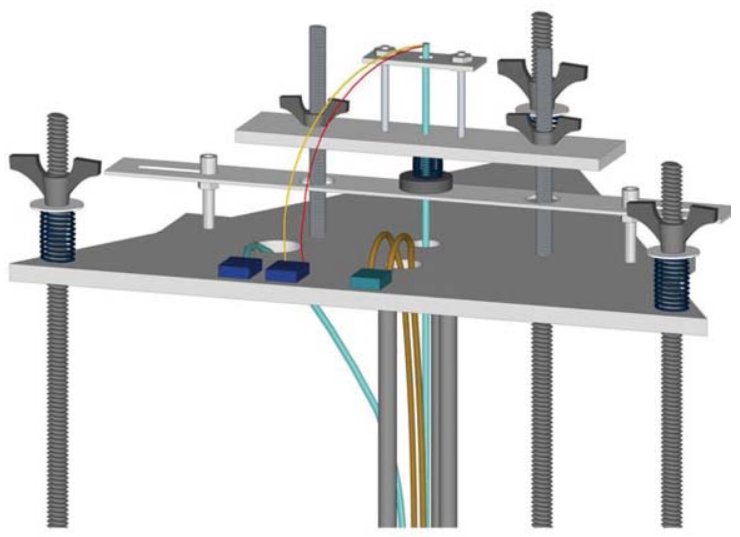

FIG. 4. (Color online) Schematic diagram of the uniaxial 4-point Seebeck instrument. (a) 2D schematic with description of labels in text, (b) complete 3D design, and (c) top plate showing thermocouple compression assembly. 
The Seebeck coefficient should be measured during both the heating and the cooling cycle. This ensures both repeatability and confirms the sample has not changed during the heating in the measurement environment. Reliability of the data is confirmed by overlapping the Seebeck coefficient data taken during both heating and cooling. Usually, the deviation between data sets is within $\pm 5 \mu \mathrm{V} / \mathrm{K}$. Accuracy, accounting for systematic errors are difficult to estimate and will depend on the sample type and shape. Typically we estimate accuracy to be $5 \%-10 \%$ for reproducible Seebeck data.

\section{PARTICULAR EMBODIMENT}

Figure 4 shows the instrument design utilized in our laboratory. For visual simplicity, the front leg $(m)$ at the bottom of the fixture shown in Fig. 4(b) is not shown in Fig. 4(a). This uniaxial 4-point geometry employs a horizontal mirror plane through the center of the sample. The sample (a) is in contact with two boron nitride cylinders (b) which have a ring of six embedded cartridge heaters, installed parallel to the sidewall of the cylinder. This annular, symmetric heater cartridge placement around the contact area allows uniform distribution of heat to the sample. Boron nitride satisfies the need for an insulating, chemically inert contact and additionally provides high thermal conductivity. Thin walled Inconel (Special Metals Corporation Inc., New York) tubes (c) are threaded into the heater assembly (b) and connected to base-plates (d) via collar clamps. This adjustable, three-legged geometry ensures good mating between the upper and lower heater assemblies. The upper and lower base-plates are aligned through the use of a framework rod (e) at each corner. A compressive force is exerted on the base-plates, and thus sample, from springs controlled by wing-nuts (f) attached to the threaded framework rods (e). Together, assembly b-f immobilizes the sample, but is adjustable to accommodate slightly non-parallel sample surfaces and thus achieves good thermal contact between the heaters and the sample.

For the measurement of the Seebeck coefficient, long (20 $\mathrm{cm}$ ) crossed-wire thermocouples (g) travel down the axis of the instrument. The thermocouple wires are contained within a four-bore mullite tube, 1/16 inch in diameter. Mullite is chosen because it has lower thermal conductivity compared to alumina, which is a typical material for thermocouples. From the sample, the thermocouple passes through the boron nitride $(\mathrm{BN})$ heater, the base-plate and into the thermocouple compression assembly (TCA). The goal of the TCA is to provide low thermal contact resistance between the sample and the thermocouple. Attached to the thermocouple is a collar clamp (h) which provides a face for a spring (i) to contact. Adjustment of plate (j) in the center of the TCA sets the compression on this spring. The uppermost assembly (k) in the TCA provides structural support to the thermocouple in case of shearing force from the operator. When changing samples, the spring within the TCA exerts a force on the collar clamp and drives the thermocouple tip past the heater surface. Such motion exposes the thermocouple tip, which is mechanically weak and readily fractures. To avoid this motion, the lowest plate (l) on the TCA is used to compress spring (i) during sample exchange. The upper assembly shows this plate engaged, while the lower assembly shows the plate when the collar clamp is free to move. The measurement assembly is supported on three legs (m) which are sufficiently long to provide access to the lower TCA. The heaters are surrounded by reflective heat shielding (o) to prevent radiative losses. During sample exchange, the heat shielding drops onto the lower triangular base-plate.

For proportional-integral-differential temperature control, the boron nitride cylinders have embedded thermocouples (n). These thermocouples are not involved in the measurement of the Seebeck coefficient and are only used for temperature control of heaters. The resulting temperature
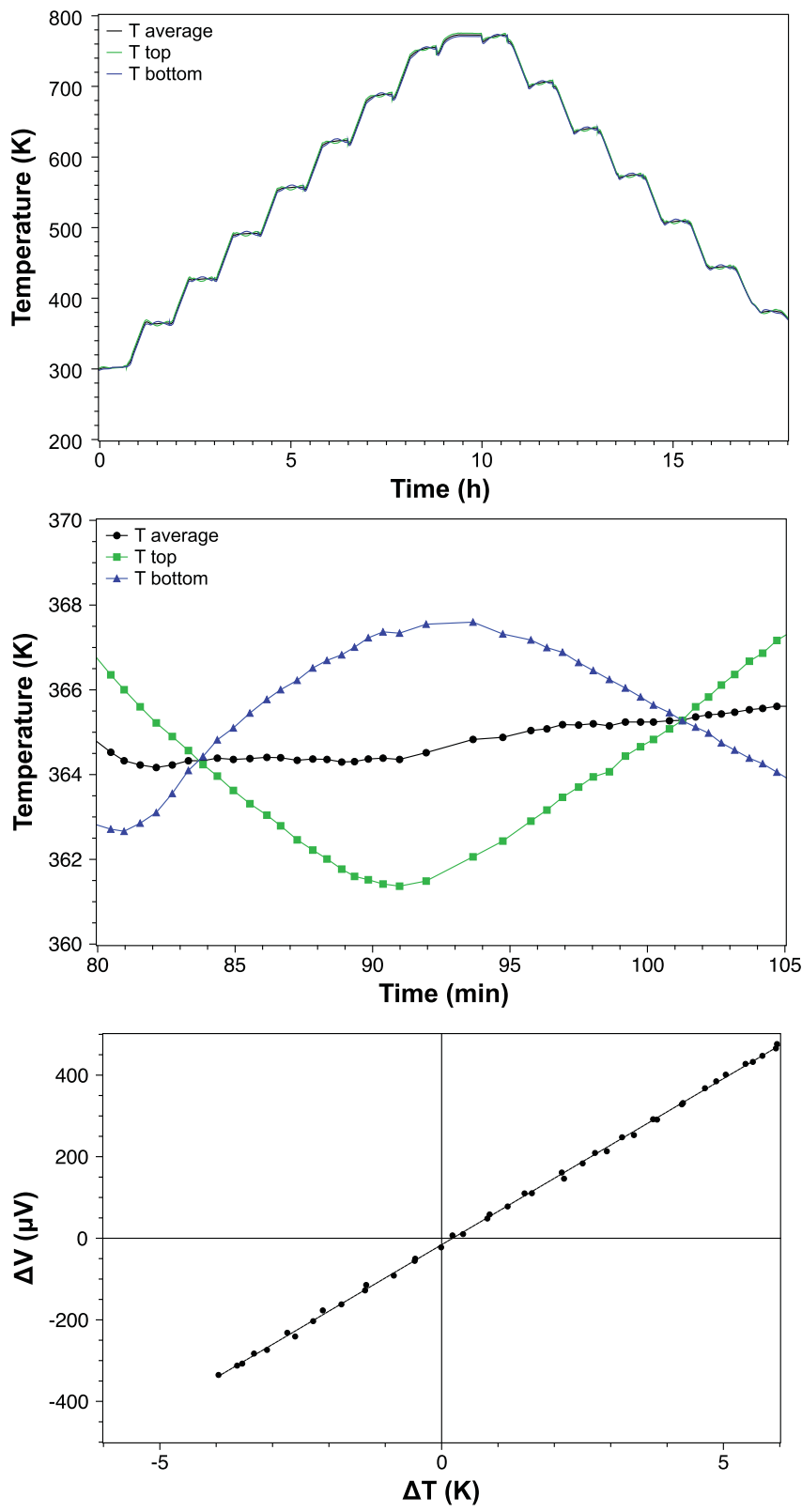

FIG. 5. (Color online) A high temperature Seebeck coefficient measurement involves temperature oscillations at many specific temperatures. (a) The top and bottom temperature of the sample along with the average value as a function of time. (b) A single measurement cycle giving a broad range of temperature gradients for each measurement point. (c) The slope of the voltage response to the temperature gradient yields the Seebeck coefficient removing the effect of any voltage offset at $\triangle \mathrm{T}=0$. 
oscillations in a high temperature run are shown in Fig. 5. The temperatures shown in panels (a) and (b) are from the Seebeck measurement thermocouples $(\mathrm{g})$. For measurements at each temperature, the temperature gradient is oscillated about a fixed average temperature, as shown in Fig. 5(b). The resulting voltage response to the temperature gradient is shown in Fig. 5(c), and reveals a linear relationship with the slope yielding the Seebeck coefficient. Representative Seebeck data can be found in a number of our publications compared to other methods. ${ }^{14,15}$

Several benefits are worth noting for this particular system design. The fixture above the horizontal mirror plane through the center of the sample, including the TCA, is assembled in one piece, and can be manually moved vertically when mounting a sample. The vertical range of movement is guided by framework rods (e) that go through the three holes located at each corner of the triangular base-plate (d). Since these holes are intentionally made much larger than the diameter of the framework rods (e), there is room for the top heater surface to be placed on a sample surface with some angle. This design flexibility allows better contact between the sample and the heaters when the sample surfaces are not exactly parallel.

Additionally, all springs are located far away from the sample heater area so that they do not lose elastic compression upon heating. This uniaxial design allows clearance around the sample heater area, and a cylindrical heat shield or clamshell furnace can be located around the sample/heater area. The heat shield ensures uniform heating near the sample area.

\section{SUMMARY}

In summary, we have reviewed the traditional designs for Seebeck coefficient measurement systems and identify measurement issues that can lead to systematic errors. To overcome these issues, a uniaxial 4-point method and other considerations such as thermocouple design are implemented in a custom built measurement system. The facile and flexible design also maximizes the measurement accuracy and has enabled rapid and reproducible data collection.

\section{ACKNOWLEDGMENTS}

The authors thank Danny Zoltan and funding by NASAJPL, and ARPA-E.

${ }^{1}$ C. P. Poole, H. A. Farach, R. J. Creswick, and R. Prozorov, Superconductivity, 2nd ed. (Elsevier, Amsterdam, The Netherlands, 2007), pp. 516-522.

${ }^{2}$ J. Prakash, S. J. Singh, S. Patnaik, and A. K. Ganguli, J. Phys.: Condens. Matter 21, 175705 (2009).

${ }^{3}$ J. Mizusaki, J. Tabuchi, T. Matsuura, S. Yamauchi, and K. Fueki, J. Electrochem. Soc. 136(7), 2082 (1989).

${ }^{4}$ G. J. Snyder and E. S. Toberer, Nature Mater. 7, 105 (2008).

${ }^{5}$ J. Martin, T. Tritt, and C. Uher, J. Appl. Phys. 108, 121101 (2010).

${ }^{6}$ V. Ponnambalam, S. Lindsey, N. S. Hickman, and T. M. Tritt, Rev. Sci. Instrum. 77, 073904 (2006).

${ }^{7}$ C. Wood, D. Zoltan, and G. Stapfer, Rev. Sci. Instrum. 56, 5 (1985).

${ }^{8}$ I. Savija, J. R. Culham, and M. M. Yovanovich, in ASME Proceedings of InterPACK2003: International Electronic Packaging Technical Conference and Exhibition Maui, Hawaii, IPack2003-35264 (2003).

${ }^{9}$ R. B. Roberts, Philos. Mag. B 43, 1125 (1981).

${ }^{10}$ R. B. Roberts, F. Righini, and R. C. Compton, Philos. Mag. B 52, 1147 (1985).

${ }^{11}$ A. T. Burkov and M. V. Vedernikov, in Condensed Matter: Disordered Solids, edited by S. K. Srivastava and N. H. March (World Scientific, New Jersey, 1995), pp. 361-424.

${ }^{12}$ G. W. Burns, M. G. Scroger, G. F. Strouse, M. C. Croarkin, and W. F. Guthrie, Temperature-Electromotive Force Reference Functions and Tables for the Letter-Designated Thermocouple Types Based on the ITS-90, Natl. Inst. Stand. Technol. Monograph 175 (1993), p. 630.

${ }^{13} \mathrm{H}$. Weiss, Z. Naturforsch. A 11A, 131-138 (1956).

${ }^{14}$ Y. Pei, A. LaLonde, S. Iwanaga, and G. J. Snyder, "High thermoelectric figure of merit in heavy hole dominated PbTe," Energy Environ. Sci. 4, 2085 (2011)

${ }^{15}$ Y. Pei, J. Lensch-Falk, E. S. Toberer, D. L. Medlin, and G. J. Snyder, Adv. Funct. Mater. 21, 241 (2010). 